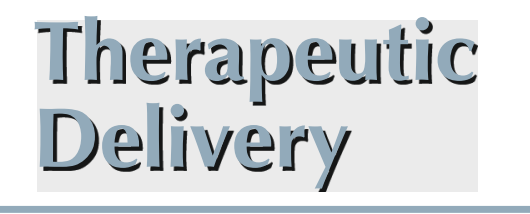

\title{
Oleogels: a promising tool for delivery of hydrophobic bioactive molecules
}

\author{
Seeking to achieve desired functional benefits in humans, \\ molecular delivery systems provide the means for direct \\ administration of bioactive molecules.
}

\section{Submitted: 1 October 2015; Accepted: 12 October 2015; Published online: XXX}

Gels can be found in a wide range of applications; from cosmetics and pharmaceuticals to food products. By 'gels' one refers to semisolid materials composed of a liquid phase, which is stabilized by an expansive mesh network composed of self-assembled gelator molecules. Two primary types of gels are found in the literature, based on the solvent used in their formation: water-based gels, known as hydrogels, and organic-based gels, known as organogels.

Hydrogels are comprised of a highly amenable structure, which affords a way for controlling the material's physical properties, by altering the network density. This structure offers the means to load bioactive molecules into the matrix, a process, which in turn can be reversed, thereby releasing those molecules in a sustained manner under appropriate conditions. Due to their high water content, hydrogels are typically chemically and mechanically biocompatible with their respective physiological environment. They can be chemically designed to be either stable or biodegradable depending on necessity. Other advantages are found in the ability of hydrogels to either deform or conform to the shape of their target site. Despite their advantages, hydrogels suffer certain limitations, where drug delivery is concerned. The low tensile strength of many hydrogels limits their ability to bear bioactive molecules over time, while their hydrophilic nature limits the loading capacity of hydrophobic molecules onto the matrix [1]. Organogel systems, on the other hand, offer physical properties, similar to those of hydrogels, with additional unique chemical properties. Due to their hydrophobic nature, organogels can dissolve higher amounts of hydrophobic bioactive molecules, subsequently delivering those molecules through different physiological routes [2]. The extensive amount of hydrophobic bioactive molecules in pharmaceutical, cosmetic and food-related applications, has caused the interest in organogels as carriers for such molecules to grow rapidly. In addition to these benefits, the lack of aqueous media in organogels deters microbial growth, hence expanding shelf life and possible future inflammation processes [3].

Organogels can be classified into two major groups depending on the gelator component used. One considers low molecular weight organogelators (LMOGs), as well as high molecular weight organogelators (HMOGs). LMOGs are comprised of a hierarchical assemblage of gelator molecules, whose bonds are facilitated by weak physical molecular interactions. Those assemblages form 3D architectures such as tapes, rods, fibers and sheets. The molecular interactions within are based on: hydrogen bonding, $\pi-\pi$ stacking, van der Waals forces, electrostatic interactions, dipole forces, hydrophobic forces and London dispersion forces [3]. The gelation mechanism is based on aggregation processes governed by either molecular selfassembly or crystallization, both instigated by external conditions such as temperature and shear. These aggregation processes depend upon a delicate balance between the gelator's solubility and insolubility in a given chemical environment; for example, solvent characteristics, temperature and so on [2]. The three-dimensional LMOG network

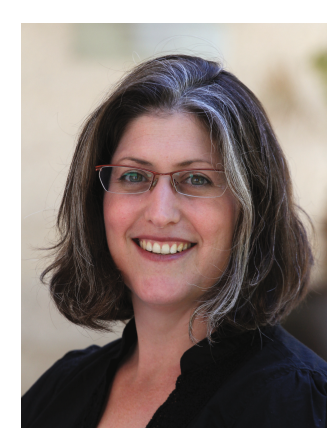

Maya Davidovich-Pinhas Faculty of Biotechnology \& Food Engineering, Technion - IIT, Israel Tel.: +972 48293346 dmaya@tx.technion.ac.il 
acts as a skeleton, and confers strength and resilience to the organogel [4]. Hence, its physical properties are strongly effected by geometric parameters of the primary structurant; for example, surface area and volume, parameters which are directly affected by cooling rate and shearing during gelation. HMOGs are primarily polymer based gels formed either by chemical reactions or physical interactions, each facilitating the supramolecular network [5]. HMOGs formed by chemical reactions are irreversible in nature, their physical properties strongly affected by the cross-linking density. HMOGs facilitated by physical interactions form a reversible dynamic network, which offers a unique way to alter the material's properties through external stimuli such as temperature and shearing. Both systems are strongly affected by the molecular weight of the polymer backbone $[5,6]$.

Despite the abundance and variety of studies done with organogel systems, relatively few investigated organogels employed in drug delivery applications. Issues of biocompatibility and toxicity of the organic solvent and the organogelator used are obviously crucial in this context. Thus far, most organogel systems under consideration comprised pharmaceutically unacceptable or untested organic solvents and gelator molecules [2,4]. However, some organogel formulations consisting of generally recognized as safe (GRAS) materials were successfully used as dermal/transdermal delivery systems, and, to a lesser extent, oral, nasal, rectal, in situ and parenteral delivery routes were studied as well $[4,6]$. These formulations predominantly use oil as their organic solvent, due to its unique properties and biocompatibility with physiological environments. Such oil-based systems are referred to as oleogels rather than organogels, to emphasize the specific nature of the solvent. As in the case of organogels, the formation of oleogels, also termed oil structuring, is based on mixing a structurant agent, or gelator, with liquid oil.

Oils and fats are made up of a variety of triacylglycerol (TAG) molecules which differ in melting temperature, leading to either solid or liquid consistency in room temperature. Most oil structuring strategies in oleogel formation rely on LMOG gelators that mimic the ability of high melting temperature TAGs to selfassemble and crystallize to form an organized solid fat structure. A solid TAG fat structure comprises several levels of organization; starting from organization into lamellar structures, which are then stacked into layers that form crystal platelets, which in turn self-assemble to create a $3 \mathrm{D}$ network that makes up the entire bulk fat. In the literature, several LMOG oil gelators based on waxes, fatty acids, fatty alcohol, n-alkens, hydroxylated fatty acids, mono/di-acylglycerols, phytosterols, ceramides, lecithin and sorbitan ester can be found, all capable of self-assembling and creating crystalline 3D networks that bind the liquid oil in the mixture. Only one HMOG direct polymer oil gelator - ethyl-cellulose - capable of gelling liquid oil has been discovered to date $[7,8]$.

Seeking to achieve desired functional benefits in humans, molecular delivery systems provide the means for direct administration of bioactive molecules. Oleogel systems offer a natural way to improve the bioavailability and bioaccessibility of hydrophobic bioactive molecules during oral administration, due to the hydrophobic nature of TAG molecules. It is important to note, that the liquid oil in the mixture is also made up of TAG molecules, though of those with low melting temperature. During digestion the liquid oil in the system tends to migrate out of the gel network and disperse in the physiological aqueous phase. The liquid TAG molecules tend to create micelles in the aqueous phase, and serve as agents for dispersing the hydrophobic bioactive molecules [9]. The micellarization and solubilization of the hydrophobic bioactive molecules, within the micelles, plays an important role in determining their bioavailability [10]. The micelles, formed during digestion, spread throughout the physiological environment, serving as vehicles for the bioactive molecules. They easily cross hydrophobic barriers, such as cell walls, thereby promoting targeting and delivery of those bioactive molecules.

To conclude, the unique nature of oil-based gel systems with GRAS status structuring agents and liquid oils offers a remarkable alternative for applications that rely on delivery of hydrophobic bioactive molecules into living tissue. The properties of oleogels are malleable, they can be altered by controlling for various physical parameters during the process of formation. Such flexibility enables adjustment of a wide range of attributes, which makes oleogels potentially suitable for a variety of applications, going from pharmaceutical therapeutic treatments, through dietary supplements, all the way to bioactive delivery in cosmetics. Further research is needed in order to study oleogels with regard to the specific bioactive molecules loaded into the system, the live tissue sites targeted and the desired effects sought.

\section{Financial \& competing interests disclosure}

The author has no relevant affiliations or financial involvement with any organization or entity with a financial interest in or financial conflict with the subject matter or materials discussed in the manuscript. This includes employment, consultancies, honoraria, stock ownership or options, expert testimony, grants or patents received or pending, or royalties.

No writing assistance was utilized in the production of this manuscript. 


\section{References}

1 Hoare TR, Kohane DS. Hydrogels in drug delivery: progress and challenges. Polymer 49(8), 1993-2007 (2008).

2 Vintiloiu A, Leroux JC. Organogels and their use in drug delivery - a review. J. Controlled Release. 125(3), 179-192 (2008).

3 Skilling KJ, Citossi F, Bradshaw TD, Ashford M, Kellam $\mathrm{B}$, Marlow M. Insights into low molecular mass organic gelators: a focus on drug delivery and tissue engineering applications. Soft Matter. 10(2), 237-256 (2014).

4 Murdan S. Organogels in drug delivery. Exp. Opin. Drug Deliv. 2(3), 489-505 (2005).

5 Suzuki M, Hanabusa K. Polymer organogelators that make supramolecular organogels through physical cross-linking and self-assembly. Chem Soc Rev. 39(2), 455-463 (2010).
6 Sagiri SS, Behera B, Rafanan RR et al. Organogels as matrices for controlled drug delivery: a review on the current state. Soft Materials 12(1), 47-72 (2014).

7 Co ED, Marangoni AG. Organogels: an alternative edible oil-structuring method. J. Am. Chem. Soc. 89(5), 749-780 (2012).

8 Marangoni AG, Garti N. Edible oleogels: structure and health implications. AOCS Press, Urbana, IL, USA (2011).

9 Marze SB. Bioaccessibility of nutrients and micronutrients from dispersed food systems: impact of the multiscale bulk and interfacial structures. Crit. Rev. Food Sci. Nutr. 53(1), 76-108 (2013).

10 Wright AJ, Pietrangelo C, MacNaughton A. Influence of simulated upper intestinal parameters on the efficiency of beta carotene micellarisation using an in vitro model of digestion. Food Chem. 107(3), 1253-1260 (2008). 